\title{
La Mammomonogamose des ruminants domestiques et sauvages
}

\author{
par M. GRABER, J. EUZEBY, J. GEVREY, P. M. TRONCY, \\ J. THAL $\left({ }^{*}\right)$
}

RESUME

Les auteurs font le point des connaissances actuelles sur la Mammomonogamose des ruminants domestiques et sauvages, maladie qui touche le bœuf, le buffle, le zébu, le mouton, la chèvre, des cervidés sud-américains, des bovidés et des giraffidés africains, ainsi que l'homme dans les zones d'endémicité (Asie du Sud-Est; Antilles et Amérique du Sud).

Ils redécrivent les deux espèces principales: Mammomonogamus laryngeus et Mammomonogamus nasicola.

Ils insistent sur la nécessité de procéder systématiquement en milieu tropical chaud et humide à des examens coproscopiques et à des autopsies complètes intéressant les voies aériennes supérieures et les cavités nasales qui sont les points de fixation habituels des Mammomonogamus.

La Mammomonogamose est une affection parasitaire de l'appareil respiratoire due à la présence dans les cavités nasales, le larynx, le pharynx, la trachée et les bronches de Nématodes appartenant à la famille des Syngamidés (sous-famille des Syngaminés) et au genre Mammomonogamus, tel qu'il a été défini par RYZHIKOV (1948), à savoir : $M$. laryngeus (RAILLIET, 1899); $M$. nasicola (VON LINSTOW, 1899) et $M$. Okapiae (VAN DEN BERGHE, 1937).

Elle frappe un grand nombre de ruminants domestiques et sauvages.

C'est une maladie des pays tropicaux chauds et humides peu connue, sporadique et discrète dans ses manifestations cliniques.

Jusqu'à présent, elle était considérée comme une helminthose mineure, sans grande incidence économique.

Cependant, au cours de ces dernières an-

(*) Institut d'Elevage et de Médecine vétérinaire des Pays tropicaux, Laboratoire de Farcha, B.P. no 433; Fort-Lamy, République du Tchad; Laboratoire de Parasitologie, Ecole nationale vétérinaire de Lyon, France. nées, de nouveaux cas de syngamose des ruminants ont été observés en Afrique, en Asie, en Amérique centrale et en Amérique du Sud.

Il a paru intéressant de faire le point de la situation actuelle, d'autant plus que, dans certaines régions du globe, Mammomonogamus est capable d'infester accidentellement l'homme.

\section{MAMMOMONOGAMOSE DES RUMINANTS}

\section{LES ESPECES EN CAUSE. LEUR DESCRIPTION}

\subsection{Les espèces}

Actuellement, on connaît trois espèces de Syngames parasites des ruminants domestiques et sauvages :

- Mammomonogamus okapiae (VAN DEN BERGHE, 1937) recueilli dans les bronches de l'Okapi. Cette espèce a été redécrite par BAER en 1950, à partir du matériel d'ori- 


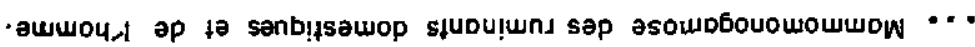

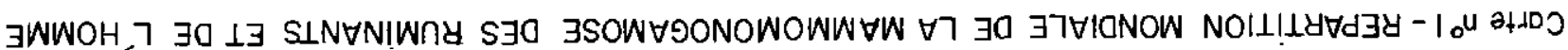

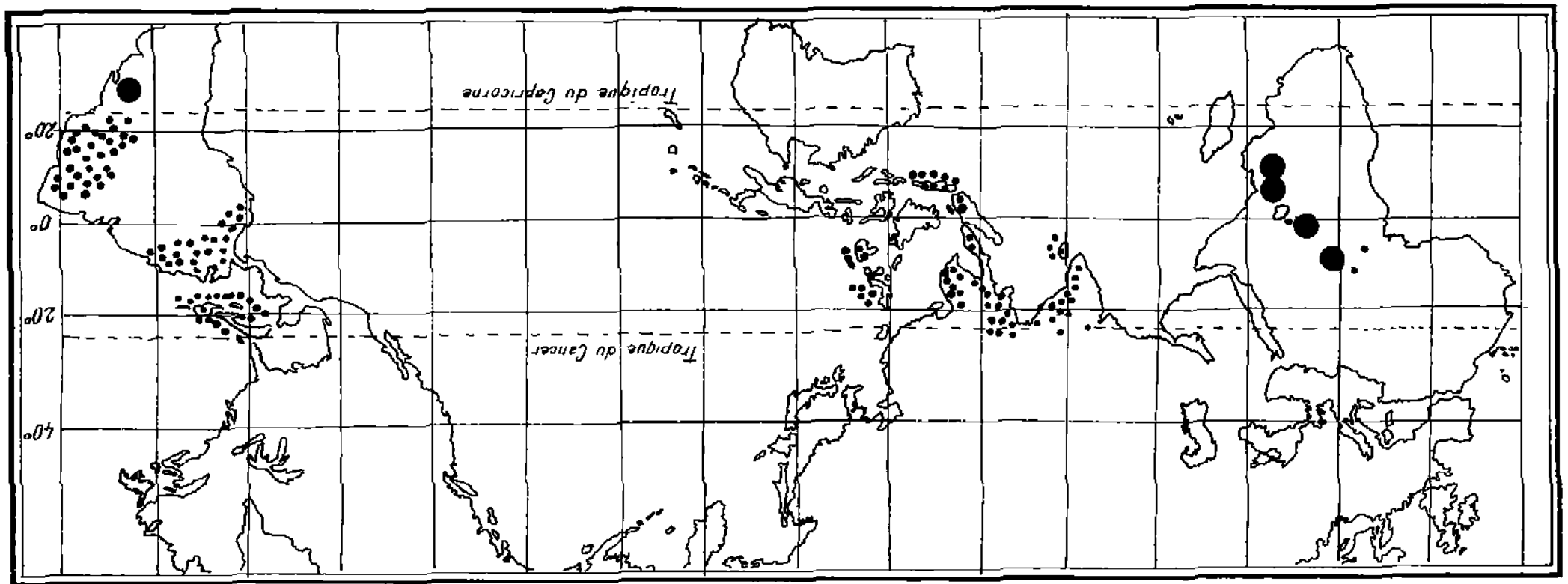


gine passablement macéré et gonflé dans le chloral et la glycérine.

- Mammomonogamus laryngeus (RAILLIET, 1899), (= S. laryngis, Lingard, 1899; $M$. bovis, Wallach; $M$. laryngeus minor et major, Smit 1922).

- Mammomonogamus nasicola (VON LINSTOW, 1899).

Les deux dernières, très proches l'une de l'autre, ont été mises en synonymie par RYZHIKOV (1948) et SKRJABIN et collab. (1961) sous le nom de Mammomonogamus laryngeus. Cette opinion est loin d'être partagée par tous les auteurs (NEVEU-LEMAIRE, 1936; YAMAGUTI, 1961; SOULSBY, 1965; GRETILLAT, 1966; LAPAGE, 1967; LEVINE, 1968).

A l'occasion de l'examen d'un couple de Syngames découvert dans la trachée d'un jeune buffle (Syncerus caffer aequinoxialis) de quatre ans abattu le 23 juin 1970 à $60 \mathrm{~km}$ de $\mathrm{Dji}$ (République Centrafricaine), nous avons été amené à revoir les caractères morphologiques différentiels des Syngames de ruminants. Pour ce faire, outre la paire précédente, ont été examinés 10 couples de Mammomonogamus laryngeus (Prototypes de RAILLIET - Collection de l'Ecole Nationale vétérinaire d'Alfort) et 10 couples de Mammomonogamus nasicola de la Collection MAROTEL (Ecole Nationale Vétérinaire de Lyon). Les résultats (GRABER, EUZEBY, GEVREY et TRONCY) montrent que $M$. laryngeus et $M$. nasicola sont bien deux espèces différentes, sous réserve d'une confirmation ultérieure par des études précises de biologie.

\subsection{Description}

Les syngames de ruminants sont des Nématodes dont la coloration est rouge vif ou blanc marbré et dont la cuticule est striée, surtout dans la région antérieure du corps. Ils sont constamment accouplés. Le mâle est fixé presque à angle droit au quart antérieur de la femelle où se trouve l'orifice vulvaire. La taille des mâles étant sensiblement inférieure à celle des femelles, les extrémités céphaliques du couple sont à peu près au même niveau, ce qui lui donne un aspect caractéristique en $Y$, d'où le nom de "vers à deux têtes" ou de "vers fourchus" qui leur a été parfois donné.

L'extrémité antérieure du corps en vue apicale a l'aspect suivant : le bord de la capsule buccale est découpé en six endroits différents délimitant six festons inégaux dont les plus longs sont en position ventrale et dorsale et les quatre autres en position latérale. Il existe six papilles céphaliques difficiles à voir, deux latérales, deux subventrales et deux subdorsales, très proches de l'ouverture buccale et qui correspondent aux six échancrures de la capsule.

Celle-ci, en vue latérale ou antéro-postérieure, est petite, épaisse, cupuliforme ou cylindro-conique (Planche $\mathrm{n}^{\circ} \mathrm{I}$, figures 1 et 2 ). Ses dimensions figurent au tableau $n^{\circ}$ I. A sa base, se trouvent huit dents triangulaires, saillantes, disposées en cercle et plus ou moins éloignées les unes des autres. De ces dents, partent des côtes chitineuses qui maintiennent l'écartement et la rigidité de la capsule buccale. Chez Mammomonogamus laryngeus (Planche I, figure 2), six d'entre elles (deux latérales, deux subdorsales et deux subventrales) atteignent le bord antérieur de la capsule qu'elles divisent en six festons; les deux dernières, la dorsale et la ventrale, s'arrêtent à mi-chemin ou au-delà. Chez Mammomonogamus nasicola (Planche I, figure 1), seules la côte dorsale et les deux subventrales sont complètes et longent la paroi de la capsule de bout en bout, les autres se terminant à des hauteurs variables (moitié ou deux tiers).

La capsule buccale est suivie d'un œsophage en forme de massue. Les papilles cervicales arrondies sont, en général, situées au voisinage de l'anneau nerveux, c'est-à-dire à peu de distance du milieu de l'œsophage (Planche I, figure 1).

Le mâle dont la taille varie selon les espèces (Tableau $\mathrm{n}^{\circ} \mathrm{I}$ ) est un peu plus épais en arrière qu'en avant. La bourse caudale est peu développée. Elle est soutenue par des côtes ventrales souvent réunies en un tronc unique et les côtes latérales très proches les unes des autres, la médio-latérale étant la plus courte. La côte externo-dorsale, mince, naît séparément de la dorsale. Celle-ci, fendue sur une partie de sa longueur, se termine par deux branches dont l'extrémité est simple $(M$. laryngeus) ou dichotomisée (M. nasicola). Les cordons testiculaires s'étendent en arrière du milieu du corps. Les spicules longs de $25-30 \mu$ (CHAPIN, 1924) ont la forme de fins bâtonnets qu'il est difficile, voire impossible, de mettre en évidence $\left(^{*}\right.$ ) (RAILLIET, 1899;

(*) C'est le cas de tous les mâles ayant fait l'objet de cette étude. 


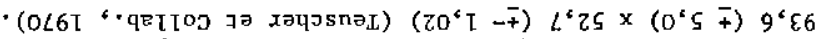

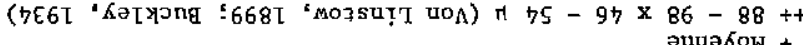

\begin{tabular}{|c|c|c|c|c|c|}
\hline $\begin{array}{l}85 \\
26\end{array}$ & $\begin{array}{l}67-27 \\
78-89\end{array}$ & $\begin{array}{l}\left(9^{\circ} 95\right) 8^{\circ} 09-\varepsilon^{\circ} 15 \\
\left(L^{\circ} L 8\right) Z^{\circ} 16-8^{\circ} 6 L\end{array}$ & $\begin{array}{r}7 \zeta-L^{\circ} \varepsilon \eta \\
++^{86}-9^{\circ} \varsigma L\end{array}$ & $\begin{aligned}(I \dagger) & L^{\prime} \varepsilon \dagger-8 \eta \\
\left(L^{\prime} 6 L\right) & S^{\prime} \varsigma 8-9 L\end{aligned}$ & 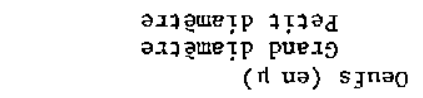 \\
\hline- & - & $\eta^{\prime} Z \subseteq \zeta$ & $56 \varepsilon-06 \mathrm{I}$ & $\left(Z^{\circ}+0 Z\right) L^{\circ} 9 \varepsilon z-E^{\prime} \varepsilon \angle I$ & ( $h$ uə) ənanb - snue әวuejsta \\
\hline- & - & $\begin{array}{l}00^{\circ} \mathrm{T} \\
28^{\circ} 0\end{array}$ & $\begin{array}{l}7^{6} \mathrm{~L}-76^{\circ} \mathrm{O} \\
Z^{\circ} \mathrm{L}-8^{\circ} 0\end{array}$ & $\begin{array}{l}\left(\varepsilon 8^{\circ} 0\right) 06^{\circ} 0-08^{\circ} 0 \\
\left(9 L^{\circ} 0\right) Z 8^{\circ} 0-Z L^{\circ} 0\end{array}$ & 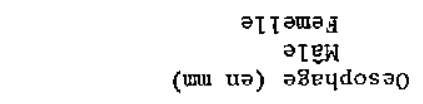 \\
\hline $\begin{array}{l}057 \\
005 \\
729 \\
075\end{array}$ & $\begin{array}{r}Z I \dagger-S I E \\
00 \varepsilon \\
\angle I S-87 \varepsilon \\
09 \varepsilon\end{array}$ & $\begin{array}{l}\text { O8E } \\
\text { ozE } \\
085 \\
0 Z 7\end{array}$ & $\begin{array}{l}097-02 \varepsilon \\
08 \varepsilon-082 \\
079-257 \\
087-51 \varepsilon\end{array}$ & $\begin{array}{l}\left(\eta^{*} \eta \zeta \varepsilon\right) \text { OSE - OEE } \\
(L ' Z O E) \text { OZE - OLZ } \\
(I \varepsilon \eta) \text { OOS - OLE } \\
(9 T E) S S E-08 Z\end{array}$ & 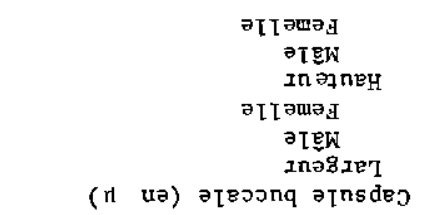 \\
\hline- & - & $8 I^{\circ} 0$ & $O E^{\prime} O-z z^{\circ} O$ & $\left(\eta \tau^{\prime} 0\right) \tau \varepsilon^{\circ} 0-\eta L^{\circ} 0$ & 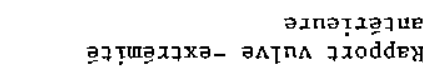 \\
\hline $\begin{array}{l}- \\
-\end{array}$ & - & $\begin{array}{l}008 \\
085\end{array}$ & $\begin{array}{l}0 \angle 8-50 L \\
005-507\end{array}$ & $\begin{array}{l}(\varepsilon 6 \varsigma) 099-07 \varsigma \\
(z 8 \varepsilon) 097-07 \varepsilon\end{array}$ & 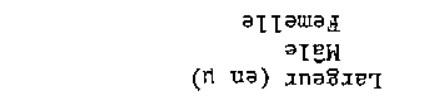 \\
\hline $\begin{array}{l}\varsigma \tau \\
\varsigma\end{array}$ & $\begin{array}{l}8^{\circ} 8 I-\nabla^{\circ} 0 I \\
\nabla^{\circ} E-I^{\prime} Z\end{array}$ & $\begin{array}{l}90^{\circ} 5 I \\
29^{\circ} \varepsilon\end{array}$ & $\begin{array}{l}\varsigma^{\prime} \varepsilon \tau-\eta^{*} L I \\
\varepsilon^{\prime} 9-\eta^{*} \varepsilon\end{array}$ & 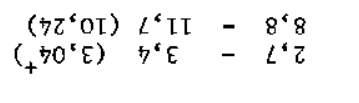 &  \\
\hline xәвg & จบุ8ิxәด्व $\operatorname{tap} \cdot \Lambda$ & \multirow{2}{*}{ 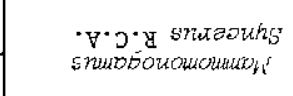 } & \multirow{2}{*}{$\begin{array}{c}\text { provzswu } \\
\text { snutofonoutumant }\end{array}$} & \multirow{2}{*}{ 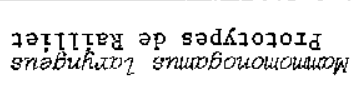 } & \\
\hline 2020 & p6onououtuon & & & & \\
\hline
\end{tabular}

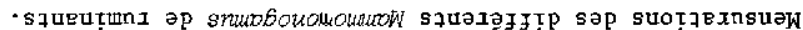




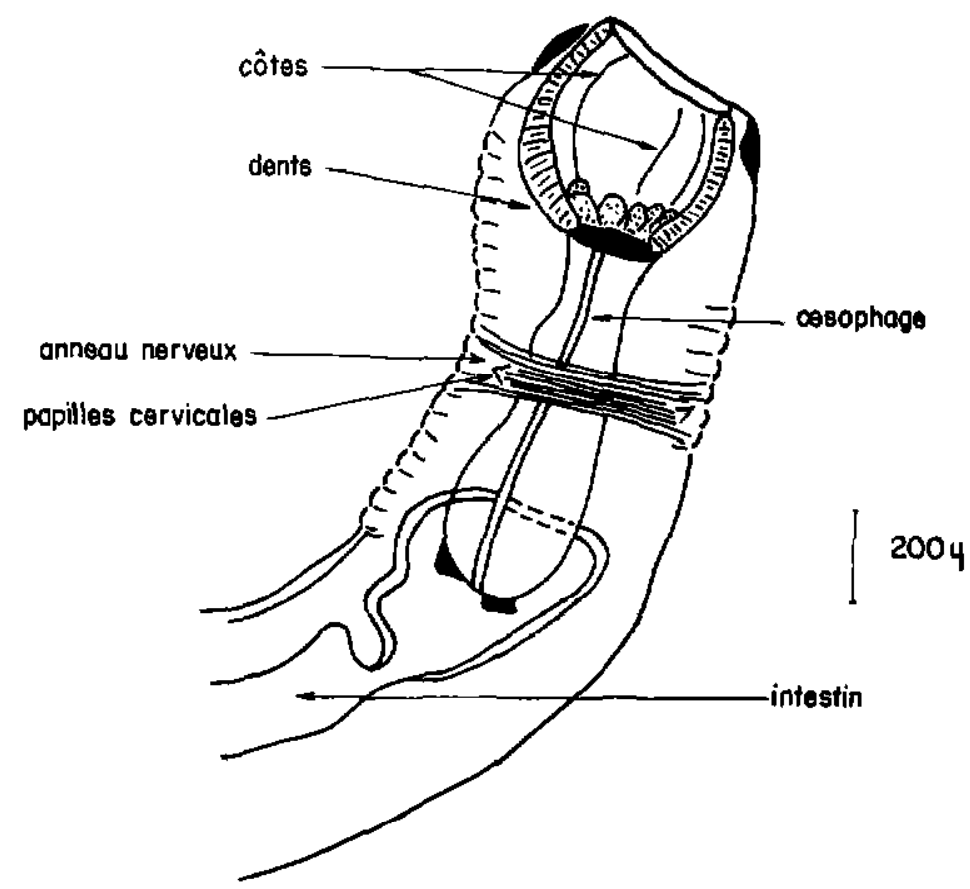

fig 1: Mâle de Mammomonogomus nosicolo (Syncerus caffer.R.C.A) Extrémité ontérieure . Vue dorso-ventrale



fig2: Capsule buccole du mâle de Mammomonogamus laryngeus (Collection Railliet)

PLANCHE No I. 
BUCKLEY, 1934) : de ce fait. ils ne peuvent servir à la diagnose des espèces, comme le suggère BHALERAO (1935).

Le corps de la fernelle (pour les mensurations, voir le tableau $n^{0} \mathrm{I}$ ), tronqué à l'extrémité antérieure, s'amincit progressivement à partir de la vulve. Le rétrécissement est particulièrement marqué dans la région anale. L’appareil génital est de type prodelphe. La longueur des utérus varie selon les espèces et les parasites. Les tubes ovariens s'allongent quelquefois fort loin dans la région postérieure, sans jamais atteindre l'anus. La queue de $M$. laryngeus (Tableau $\mathbf{n}^{\circ} \mathbf{I}$ ) est courte, obtuse et porte de nombreuses stries très fines. Celle de $M$. nasicola, est un peu plus longue et elle n'est pas striée.

Les oufs (Planche II, figures 1,2 et 3), dans l'ovojecteur et la terminaison de l'utérus, sont ellipsoïdes et non operculés. Ils sont entourés d'une coque épaisse (4 à $4,6 \mu$, d'après TEUSCHER et collab. 1970), jaunâtre, légèrement rugueuse, parfois striée, renfermant deux blastomères qui la remplissent incomplètement. Sur un total de 108 œufs examinés, ces caractères sont rigoureusement constants, quelle que soit l'origine du parasite: seules, les dimensions changent (Tableau $n^{\circ} I$ ). Ces observations sont en accord avec celles de BUCKLEY (1934), de TEUSCHER et collab. (1970) et de HO THI SANG et collab. (1970), mais ne correspondent pas à celles de WARE (1925), de PATNAIK (1963), de LEVINE (1968) et de CRAIG et FAUST (1970) : pour ces auteurs, l'œuf de Mammomonogamus laryngeus, au moment de la ponte, comporte de 4 à 8 blastomères, alors que, pour les prototypes de RAILLIET, il n'en comprend que deux. En réalité, cette différence n'est qu'apparente et n'a pas grande signification en matière de diagnose différentielle : en effet, commc nous l'avons observé à plusieurs reprises et comme cela a été signalé à propos d'Haemonchus et d'Ostertagia (Dr GEVREY, communication personnelle), les aufs de Nématodes sont susceptibles, dans certaines conditions, de poursuivre leur segmentation in utero pendant un temps limité. Tout dépend de la façon dont les „parasites sont récoltés et conservés (*).

(*) Selon HO THI SANG et Collab. (1970), les cufs de Mammomonogamus évoluent très vite à $22^{\circ} \mathrm{C}: 6$ à 10 heures après l'émission, ils possèdent déjà 4 et même 8 blastomères.
Il est bon d'attirer l'attention sur le fait que Mammomonogamus laryngeus, si l'on se réfère aux prototypes de RAILLIET, est un Syngame de petite taille dont la longueur est comprise entre $2,7 \mathrm{~mm}$ (Mâle) et $11,7 \mathrm{~mm}$ (Femelle), ce qui est confirmé par HALL (1915) et BUCKLEY (1934). Cependant, WARE (1925), DROZDZ et MALCZEWSKI (1959), PATNAIK (1963) donnent des dimensions nettement supérieures : 6,5 - 22,6 mm (Femelle); 2,5 - 5,5 mm (Mâle) et, devant cette situation, SMIT (1922) a cru bon de créer deux sousespèces, $M$. laryngeus minor et $M$. laryngeus major. Les descriptions étant souvent incomplètes, il est bien difficile de savoir si l'on a toujours affaire à Mammomonogamus laryngeus.

Quant au Syngame de Syncerus caffer de R.C.A., de par ses dimensions (Tableau $\mathrm{n}^{\circ} \mathrm{I}$ ) et ses caractères morphologiques, il s'apparente étroitement à Mammomonogamus nasicola: seule, la vulve est en position plus antérieure. L'examen n'ayant porté que sur un couple, il est difficile de tirer des conclusions définitives. Le Nématode sera donc, pour l'instant, assimilé à $M$. nasicola.

\section{REPARTITION GEOGRAPHIQUE. ESPECES ANIMALES AFFECTEES ET TAUX D'INFESTATION}

\subsection{Mammomonogamus laryngeus et Mammomonogamus nasicola}

Les deux Syngames ayant été souvent confondus, il est difficile de donner leur répartition exacte. Les indications qui vont suivre n'ont donc qu'une valeur relative.

2.1.1. Mammomonogamus laryngeus a été surtout rencontré dans le sud-est asiatique chez le buffle domestique (Buffelus bubalus), le zébu (Bibos indicus), le bœuf (Bos taurus) et, d'après DE DOES (1907), chez le cheval et chez le chien (?). Les pays les plus touchés sont :

— Les îles Philippines (HALL, 1915). 68 p. 100 des bovins de 3 à 5 ans sont porteurs de parasites à l'abattoir (Z. DE JESUS, 1938).

- l'Indonésie, l'île de Java principalement (DE DOES, 1907; SMIT, 1922; RESSANG et collab. (1959).

- Le Vietnam (RAILLIET, 1925; HOUDE- 



4

PLANCHE No II.

Fig. 1 et 2: CEufs de Mammononogamus (collection Railliet).

F1g 3 et 4 : Eufs de Mammomonogamus nasicola (collection Marotel).

Fig. 5 et 6: CEufs de Mammomonogamus nasicola (Syncerus caffer. R.C.A.). 
MER, 1938; DROZDZ et MALCZEWSKI, 1967) : M. laryngeus est largement répandu, tant dans le Nord du pays (HOUDEMER et collab. 1923) que dans le sud d'où provenaient les prototypes décrits par RAILLIET en 1899, prototypes recueillis par CARRE et FRAIMBAULT à l'Institut Pasteur de Nha-Trang (taux d'infestation, 50 p. 100).

- La Malaisie (BUCKLEY, 1934; ORR, 1938).

- La Birmanie (BHATTACHARJEE, 1937).

- Ceylan où SENEVIRATNA et MAHA LINGAM (1962) ont noté à l'abattoir de Kandy deux cas de mammomonogamose sur 62 animaux autopsiés.

- Les Indes. Les premiers cas ont été observés par LINGARD en 1898-1899 (cité par FROGER, 1930), puis par SHEATHER et SHILSTON (1920): le pourcentage d'animaux infestés est de 13 p. 100 chez les bovidés vivant en altitude et de 15 p. 100 chez les animaux de plaine. Depuis, les enquêtes se sont multipliées et, présentement, la syngamose du buffle et du bœuf est endémique au Pendjab (WARE, 1925), dans les états de Madras (MUDALIAR et ALWAR, 1947), d'Orissa avec 40 p. 100 d'animaux atteints (PATNAIK; 1963), de l'Uttar Pradesh (Kumaon et collines de Gahrwal - BAYLIS, 1936) et en Assam (ENDREJAT, 1964) où la maladie semble plus fréquente chez le buffle (15-60 p. 100 que chez le beuf (16-20 p. 100).

2.1.2. Mammomonogamus nasicola est plutôt une espèce africaine et américaine :

- En Afrique, la présence du parasite est connue depuis le début du siècle grâce à la description originale de VON LINSTOW (1899) à partir d'exemplaires prélevés dans les cavités nasales d'une chèvre autopsiée à Yaoundé (République fédérale du Cameroun). Le même parasite a été revu en 1970 chez Syncerus caffer dans l'est de la République centrafricaine.

La mammomonogamose doit également exister au Tchad, car, dans un nombre de cas très limité, des oufs de Syngames à deux blastomères ont été mis en évidence par l'un d'entre nous lors d'examens coprologiques effectués sur de jeunes zébus. Faute d'autopsies, l'espèce en cause n'a pu être précisée.
Le problème mériterait d'être repris dans le centre et le sud du Cameroun, où des cas de parasitoses "pulmonaires" d'origine indéterminée qui pourraient être dus à $M$. nasicola ont été signalés chez les petits ruminants au cours de ces dernières années.

- En Amérique où il aurait été introduit par du bétail importé d'Afrique, le Syngame a été rencontré aux Antilles (îles de la Trinitad et de St.-Vincent) par BUCKLEY (1934). Les hôtes sont le mouton (55 p. 100 d'ovins parasités), le bœuf (15 p. 100 et la chèvre. $M$. nasicola a, en outre, été trouvé au Brésil (Rio Grande Do Sul) dans le larynx de Cervus rufus (VON LINSTOW, 1899).

2.1.3. Mammomonogamus désignés sous le nom de $M$. laryngeus et qui seraient en réalité des $M$. nasicola.

Les recherches de GRETILLAT (1966) aux Antilles françaises (Guadeloupe et Martinique) et de TEUSCHER et collab. (1970) en Equateur amènent les auteurs à mettre en doute, sur le continent américain, l'existence de $M$. laryngeus, espèce assez largement répandue chez les bovidés de Porto-Rico (RANSOM, 1921), du Brésil (PINTO et ALMEIDA, 1937; BAKER, 1951; J. DA SILVA, 1961), du Vénézuela (CABALLERO et VOGELSANG, 1950) et de Colombie (GIOVINE, 1932). Se basant sur certains caractères morphologiques et sur les localisations nasales de quelques exemplaires, GRETILLAT et TEUSCHER pensent qu'il s'agit plutôt de $M$. nasicola, hypothèse qui demande à être vérifiée.

La mammomonogamose touche 40 à 60 p. 100 des bovins à la Guadeloupe et à la Martinique.

\subsection{Mammomonogamus okapiae}

C'est une espèce strictement africaine qui se superpose à l'aire de répartition de l'Okapi, c'est-à-dire l'est de la cuvette congolaise (Oubanghui, Uellé, Ituri, Kivu, région de Kinsangani).

\subsection{Autres Mammomonogamus des ruminants}

Des Syngames, non encore identifiés, ont été découverts dans les cavités nasales du Waterbuck (Kobus defassa), du Puku (Kobus vardoni), du Kob de l'Uganda (Kobus kob) et du 
Buffle (Syncerus caffer) par SACHS et SACHS (1968) et SACHS et DEBBIE (1969).

Les lieux de récolte sont: le parc de Serengeti dans le nord de la Tanzanie, la zone du lac Rukwa dans le sud et la réserve de chasse d'Aswa Lolim dans le nord-ouest de l'Uganda.

Ce sont tous des Nématodes de grande taille : $24-33 \mathrm{~mm}$ sur $0,9-1,2 \mathrm{~mm}$ chez la femelle et $6-8 \mathrm{~mm}$ sur $0,5 \mathrm{~mm}$ chez le mâle (SACHS, FRANK et BINDERNAGEL, 1969).

Mammomonogamus sp. existerait aussi en Uganda chez le bœuf (BWANGAMOI, 1968).

\section{CYCLE EVOLUTIF. MODE D'INFESTATION. RECEPTIVITE}

\subsection{Cycle évolutif et mode d'infestation}

Il n'est que très partiellement connu. On sait (BHATTACHARJEE, 1966; HO THI SANG, JUNOD et PHILBERT, 1970) que l'évolution de l'œuf pondu dans le milieu extérieur exige certaines conditions climatiques qui sont celles des régions tropicales chaudes et humides, ce qui explique la localisation géographique des Mammomonogamus de ruminants :

- Température élevée : $26-30^{\circ} \mathrm{C}$.

- Degré hygrométrique important: plus de 85 p. 100.

Les œufs donnent alors des larves infestantes $\mathrm{L}_{\mathrm{a}}$ au bout de $9-12$ jours. Elles sont contenues dans l'œuf et enveloppées de la dépouille de la larve $L_{2}$.

Elles mesurent de $300-380 \mu$ (BHATTACHARJEE, 1966; HO TRI SANG et collab. 1970) à $548 \mu$ (SAINT-JOHN et collab. 1929) sur 16 à $33 \mu$. L'œsophage est long de 108 à $130 \mu$ et l'anus est à $32-47 \mu$ de l'extrémité postérieure. La cuticule est finement striée et les parois de la capsule buccale fortement chitinisées. La larve, peu mobile, arrondie dans la région antérieure, s'amincit progressivement en pointe mousse au niveau de la queue.

On ignore ce que devient ultérieurement la larve $L_{3}$. Le cycle pourrait - il s'agit là d'une simple présomption - être voisin de celui de Syngamus trachea des oiseaux. Dans ce cas (EUZEBY, 1963), la larve $L_{a}$ a plusieurs possibilités :
- Elle demeure incluse dans l'œuf.

- Elle éclot dans le milieu extérieur, où elle fait preuve d'une activité réduite : pratiquement statique, elle est, de plus, sensible à certains facteurs de destruction, notamment la dessication.

- Elle est absorbée, après éclosion, par un hôte intermédiaire facultatif, dit hôte de transport (Mollusques; Arthropodes coprophages; Annélidés). Elle s'enkyste dans le tissu musculaire où elle est capable de survivre pendant un temps assez long. BUCKLEY (1934) pense que, pour les Mammomonogamus de Mammifères, le rôle de ce vecteur facultatif est essentiel.

C'est vraisemblablement en ingérant les hôtes de transport contaminés ou, mieux, de la terre, de l'herbe ou des eaux de boisson souillées par les œufs renfermant des larves $\mathrm{L}_{3}$ ou par les larves elles-mêmes que les ruminants seraient capables de s'infester.

Chez les animaux, la fin du cycle, c'est-à-dire la phase endogène et la durée de la période prépatente, est totalement inconnue.

\subsection{Réceptivité}

Plusieurs facteurs interviennent:

- L'espèce animale : Mammomonogamus laryngeus semble plutôt être un parasite des grands ruminants domestiques (bœuf, buffle, zébu). Mammomonogamus nasicola, outre les espèces précédentes, affecte les ovins, les caprins, ainsi que plusieurs ruminants sauvages (Cervus rufus en Amérique du Sud et Syncerus caffer en Afrique) qui ont la possibilité, au cours de leurs déplacements, de disséminer les œufs de Syngames et d'infester indirectement les animaux domestiques qui utilisent les mêmes pâtures.

- L'âge : les helminthoses sont, en général, des maladies de jeunes. En milieu tropical, ce n'est pas toujours le cas et le niveau du parasitisme (par Onchocerca, Paramphistomum et Fasciola notamment) est souvent plus élevé chez les animaux dont la croissance est terminée (5 ans et plus). Pour $M$. laryngeus, il semble en être de même dans certaines régions d'Extrême Orient (Assam) où ENDREJAT (1964) fait état d'un taux d'infestation de 60 p. 100 chez le buffle adulte contre 25 p. 100 chez le bufflon. Les observations de BUCK- 
LEY (1934) et de ROSE ROSETTE (1934)

vont dans le même sens.

\section{ETUDE CLINIQUE ET ANATOMIQUE}

\subsection{Symptômes}

Ils sont extrêmement discrets, voire inexistants (THERY, 1962).

On observe, de temps en temps, des quintes de toux sèche, plus ou moins violentes et irrégulières (TEUSCHER et collab. 1970) pouvant aller jusqu'à la suffocation et l'asphyxie, lorsque les Syngames obstruent le carrefour laryngo-pharyngien, comme c'est quelquefois le cas chez les petits ruminants. La mort survient alors en moins de deux heures (ROSE ROSETTE, 1934).

\subsection{Etude anatomique}

\subsubsection{Localisation des Syngames}

Elle est intéressante à considérer, car, si les Syngames d'oiseaux se localisent à la trachée, les Syngames de ruminants semblent avoir perdu cette spécificité (THERY, 1962). On les rencontre :

- Dans les cavités nasales : c'est surtout vrai pour Mammomonogamus nasicola (VON LINSTOW, 1899; BUCKLEY, 1934; GRETILLAT, 1966) et pour les Mammomonogamus de la grande faune africaine (SACHS, FRANK et BINDERNAGEL, 1969).

- Dans le larynx, c'est-à-dire sur le bord postérieur de l'épiglotte, les cordes vocales, les ventricules latéraux, l'aryténoide, le plancher et les côtés de l'organe. C'est la localisation la plus classique de $M$. laryngeus (RAILLIET, 1899; WARE, 1925; SENEVIRATNA et MAHAIINGAM, 1962; PATNAIK, 1963).

- Dans le pharynx (BUCKLEY, 1934; BAKER, 1951).

- Dans la trachée, PATNAIK (1963) note qu'au fur et à mesure que l'on descend l'arbre respiratoire, les Syngames deviennent moins nombreux et sont à un stade de développement moins avancé.

- dans les bronches (M. okapiae).

Pour l'instant, aucun Syngame n'a été mis en évidence dans les voics respiratoires profondes (bronchioles et alvéoles pulmonaires).

\subsubsection{Leur nombre}

La plupart du temps, on compte de 2 à 10 couples fixés à la muqueuse des voies aériennes supérieures (LINGARD), mais les parasites peuvent être beaucoup plus nombreux : 68 paires (PATNAIK, 1963), 76 paires (BUCKLEY, 1934) ou même "un bouquet " (ROSE ROSETTE, 1934).

\subsubsection{Les lésions}

Les muqueuses du conduit aérifère sont le siège d'une inflammation catarrhale avec production d'un abondant mucus spumeux et blanchâtre dans lequel les Syngames sont noyés plus ou moins complètement. Leur capsule buccale, au point de fixation aspire un bourgeon muqueux, superficiel s'il s'agit de jeunes animaux, profond si l'hôte est plus âgé. $\mathrm{Au}$ voisinage immédiat, la muqueuse s'œdématie et se congestionne. Elle renferme un grand nombre d'eosinophiles et de lymphocytes. L'adème peut quelquefois être très marqué et envahir toute la région laryngo-pharyngée (BAKER, 1951).

$\mathrm{Au}$ bout d'un certain temps, les parasites se détachent et vont s'implanter ailleurs, en laissant de petits ulcères rouges punctiformes qui correspondent aux points de fixation successifs des Nématodes.

\section{ROLE PATHOGENE}

Les syngames ont une triple action:

- Spoliatrice: ce sont des vers hématophages capables d'absorber des quantités de sang parfois importantes.

- Irritative par leur mode de fixation aux muqueuses nasales, laryngées et trachéo-bronchiques et par les déplacements qu'ils effectuent. Cette action semble particulièrement nette pour les Mammomonogamus des cavités nasales et, chez l'hippopotame atteint de syngamose, on a signalé, dans les naseaux, la formation de nodules volumineux et purulents (GHESQUIERE, 1934).

- Mécanique par accumulation des Nématodes au niveau du larynx avec obstruction des voies respiratoires et asphyxie. C'est le fait surtout des petits ruminants.

Le rôle pathogène des Syngames chez les ruminants paraît, jusqu'à plus ample informé, limité. Les Mammomonogamus semblent assez 
bien tolérés par le gros bétail, à condition qu'il soit bien alimenté et en bon état d'entretien.

\section{DIAGNOSTIC}

\subsection{Ante mortem}

\subsubsection{Clinique}

Il est difficile, car, en milieu tropical, les affections de l'appareil respiratoire sont nombreuses, tant chez les bovidés que chez les ovins et les caprins.

Il faudra éviter, chez le bouf, de confondre la mammomonogamose avec la péripneumonie, la pasteurellose, la tuberculose, les bronchites banales ou parasitaires et, chez le mouton, avec les strongyloses respiratoires et l'œstrose des sinus qui est fréquente dans les pays chauds. Dans ce cas, on observe de la sinusite avec jetage, ce qui ne semble pas être le cas de la syngamose nasale des ruminants (BUCKLEY, 1934; SACHS, FRANK et BINDERNAGEL, 1969).

\subsubsection{Expérimental}

par la recherche dans les selles des aufs de Mammomonogamus qui sont caractéristiques, lorsqu'ils renferment deux blastomères (voir supra), ce qui est souvent le cas.

Les cufs de Syngames à $4-8$ blastomères (Planche III, figure 4) peuvent être confondus chez les bovins avec les oufs de Bunostomum phlebotomum et, chez les ovins et caprins, avec ceux de Gaigeria pachyscelis. II s'agit là de deux Ancylostomidés des zones tropicales chaudes et humides dont l'aire de répartition est en grande partie superposable à celle des Mammomonogamus, Le diagnostic coproscopique est malaisé.

L'œuf de Bunostomum phlebotomum (Planche III, figures 1 et 2) qui mesure 80 à $110 \mu$ sur 40 à $65 \mu$ a des bords parallèles. ce qui lui donne "grosso modo " la forme dun rectangle à angles arrondis. Sa paroi est mince. Il renferme 4 à 8 blastomères volumineux, noirâtres.

L'œuf de Gaigeria pachyscelis (Planche IIJ, figure 3) est ellipsoïde, à coque mince, long de $110-120 \mu$ sur $50-55 \mu$. Il est, la plupart du temps, pondu à un stade de segmentation avancé.

\subsection{Post-mortem}

Les Syngames, noyés dans le mucus, ne sont pas faciles à mettre en évidence. Selon RAILLIET (1899), ils apparaissent comme " de minces filets de sang coagulé " qui n'attirent pas particulièrement l'attention, d'autant plus que, bien souvent, on néglige d'ouvrir le larynx ou les cavités nasales. C'est sans doute là, en Afrique, l'explication de la relative rareté de la mammomonogamose des ruminants domestiques. Il importe donc de faire des autopsies complètes et d'examiner très attentivement les parties supérieures de l'arbre aérien.

Pour les animaux de chasse dont les trophées doivent être conservés, il n'est pas question de fendre la tête en deux, comme on le fait pour le zébu ou pour le mouton. SACHS, FRANK et BINDERNAGEL, 1969) décrivent une technique originale de prélèvement (Planche IV) qui consiste à placer le massacre dans un cuveau rempli de sérum physiologique. Le crâne repose par les cornes et par la pointe des maxillaires supérieurs sur les bords du récipient et le carrefour laryngo-pharyngé est immergé dans le liquide. On laisse reposer pendant une nuit et, le lendemain, on recueille les Syngames qui se sont détachés et sont tombés au fond du baquet. L'examen doit être effectué minutieusement, car les parasites sont souvent inclus dans les caillots sanguins et, de ce fait, risquent de passer inaperçus.

\section{PRONOSTIC}

Bénin: la maladie est rarement mortelle, sauf chez les petits ruminants.

\section{TRAITEMENT}

Pendant longtemps, on est resté désarmé. Différents traitements ont été proposés dont des pulvérisations intralaryngiennes d'une solution tiède de Salicylate de sodium à 5 p. 100 (HOUDEMER et TRAN THO-HUY, 1923).

Récemment, TEUSCHER et collab. (1970) ont obtenu des résultats satisfaisants en injectant par la voie sous-cutanée, du Tétramisole à la dose de $5 \mathrm{mg} / \mathrm{kg}$.

L'action du Thiabendazole à $100 \mathrm{mg} / \mathrm{kg}$ paraît plus irrégulière. 

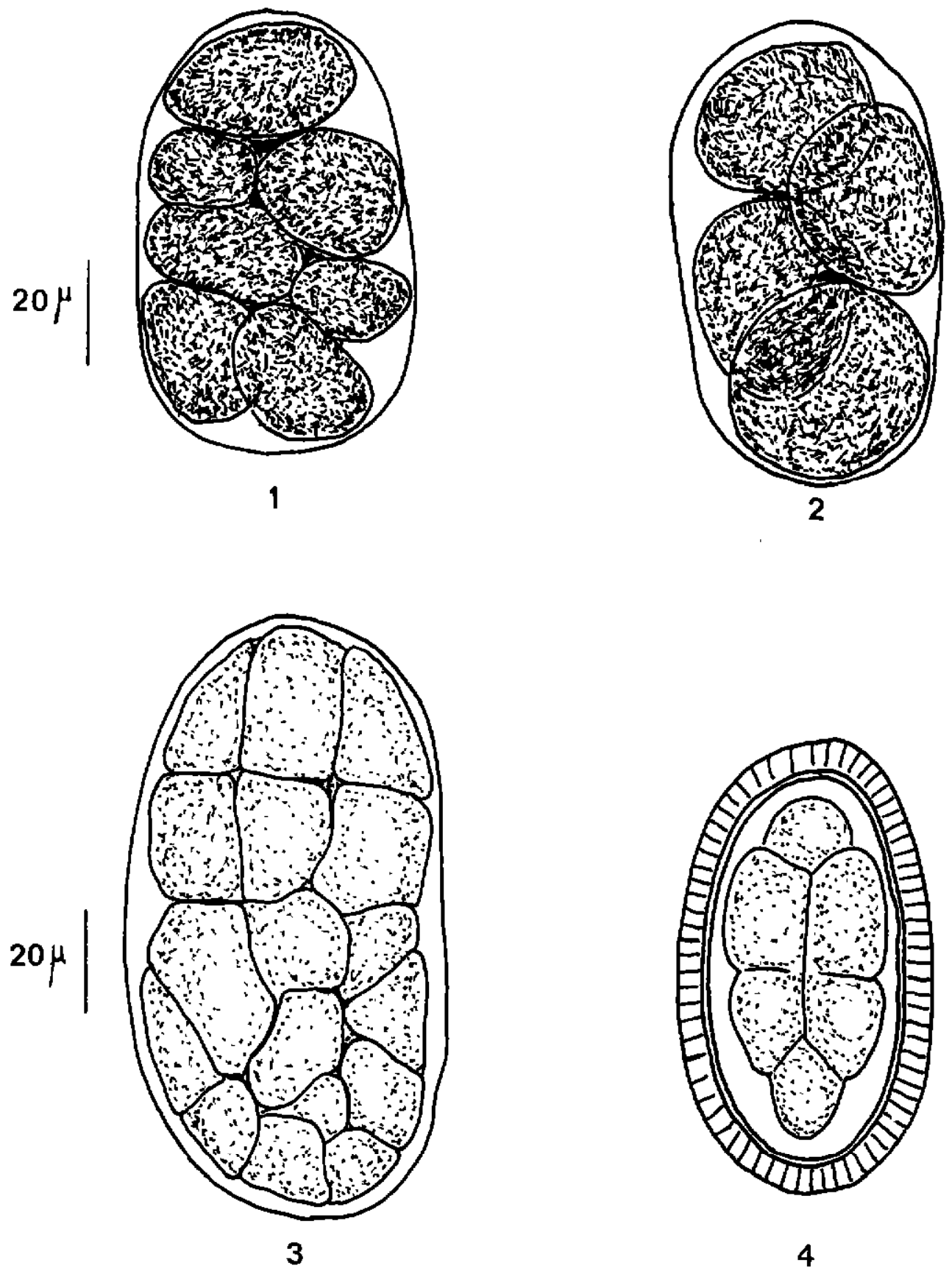

PLANCHE No III.

Fig. 1 et 2: Eufs de Bunostomum phlebotomum (zébu).

Fig. 3: Eufs de Gaigeria pachyscelis (mouton).

Fig. 4: Cûfs de Mammomonogamus laryngeus (d'après Faust, 1970). 


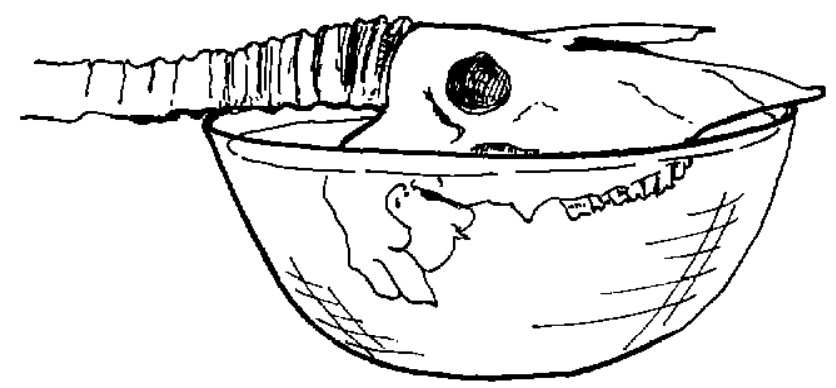

PLANCHE No IV.

Technique de récolte des Mammomonogamus de ruminants sauvages (d'après Sachs, Frank et Bindernagel, 1969).

\section{PROPHYLAXIE}

Le Cycle évolutif et le mode d'infestation étant pratiquement inconnus, il est impossible, pour l'instant, d'indiquer les règles de prophylaxie individuelle ou générale permettant de lutter efficacement contre la maladie.

Cependant, certaines précautions peuvent être prises :

- Il faudra, dans les élevages sains, éviter d'introduire des animaux porteurs de $\mathrm{Mam}$ momonogamus. Les bovins nouvellement achetés devront subir systématiquement un examen coprologique et, s'il est positif, devront être traités au Tétramisole, avant d'être mélangés au reste du troupeau.

- Il faudra empêcher les ruminants domestiques de fréquenter les mêmes parcours que les ruminants sauvages susceptibles d'héberger des Syngames. En pays de grande transhumance, c'est une mesure difficile à appliquer.

- Les pâtures et les parcs contaminés seront évacués et stérilisés (par mise en culture, feux, drainage, etc.). Les litières seront brûlées.

\section{MAMMOMONOGAMOSE HUMAINE}

C'est une affection rare et purement accidentelle due à la présence dans le larynx, le pharynx et les bronches (JUNOD, PHILBERT et HO THI SANG, 1970) de l'homme de Mammomonogamus laryngeus ou de Mammomonogamus nasicola, parasites habituels des ruminants domestiques et sauvages.
Le premier cas humain connu est celui observé par KING (1913) à Sainte-Lucie aux Antilles. LEIPER (1913-1925) décrit le Syngamus kingi. BUCKLEY (1934), réexaminant les holotypes de LEIPER met en synonymie $S$. kingi avec $S$, nasicola.

Au total, 27 cas ont été signalés depuis 1913 (Tableau $\mathrm{n}^{\circ}$ 2), dont 26 sont américains et un seul asiatique (Philippines - ST JOHN et collab. 1929). Les régions les plus atteintes sont actuellement les Antilles et le Brésil, mais d'autres zones pourraicnt l'être aussi, notamment les pays d'Afrique et d'Asie où la mammomonogamose animale est fréquente.

Les parasites en cause sont, soit Mammomonogamus laryngeus, soit Mammomonogamus nasicola. Toutefois, comme il a été dit plus haut, un doute subsiste quant à l'identité exacte des Syngames découverts chez l'homme en Amérique (TEUSCHER et collab. 1970).

La maladie dont la période prépatente serait de 25 à 40 jours (HO THI SANG, et collab. 1970) frappe surtout les adultes, plus rarement les jeunes. Les symptômes sont plus nets que chez les ruminants, ce qui s'explique par le changement d'hôte. On note une toux, sèche et persistante, en quintes, avec crachats hémoptoïques et accès de suffocation pouvant se terminer par l'expulsion d'un ou plusieurs couples de Syngames rouges et enrobés de mucus, d'où leur aspect de " filets de sang doués de mouvements " (ST-PRIX, 1950). Les crises de toux sont à prédominance nocturne : elles empêchent le sommeil avec comme conséquences : 
TABLEAU N*2

LES CAS DE SYNGAMOSE HUMAINE

\begin{tabular}{|c|c|c|c|c|}
\hline Cas & Année & Auteurs & Lieux de récolte & Parasites en cause \\
\hline 1 & 1913 & KING, cité par LEIPER & Ste-Lucie & $M$. Kingi $=M$. nasicola \\
\hline 2 & 1921 & TRAVASSOS & Brésil - Bahia & $M$. laryngeus \\
\hline 3 & 1925 & LEIPER & Guyane anglaise & M. Kingi $=M$. nasicola \\
\hline 4 & 1929 & ST JOHN et Collab. & Philippines & $M$. laryngeus \\
\hline $5-6$ & 1931 & HOFFMAN & Porto-Rico & M. laryngeus \\
\hline 7 & 1932 & HOFFMAN & Trinitad & M. laryngeus \\
\hline 8 & 1938 & MELLO et MELLO & Brésil - Rio de Janeiro & M. laryngeus \\
\hline $9-10-11$ & 1938 & ST PRIX & Martinique & M. Kingi $=M$. nasicola \\
\hline & 1939 & LENT et PENNA & Brésil - Rio de Janeiro & $M$. laryngeus \\
\hline $13-14-15$ & 1943 & FRONT & Porto-Rico & M. laryngeus \\
\hline 16 & 1945 & MONTESTRIJC et Collah. & Martinique & $M$. laryngeus \\
\hline 17 & 1947 & GOMES DE MORAES & Brésil - Rio de Janeiro & $M$. laryngeus \\
\hline 18 & 1948 & PASSOS et CASTRO BARBOSA & Brésil & $M$. laryngeus \\
\hline 19 & 1951 & WELLS & Ste-Lucie & $M$. King $i=M$. nasicola \\
\hline 20 & 1952 & LIMA et BARBOSA & Brésil - Récife & $M$. laryngeus \\
\hline 21 & 1954 & AMARAL et Collab. & Brésil & $M$. laryngeus \\
\hline 22 & 1962 & MACHADO DE MENDOCA & Brésil & $M$. laryngeus \\
\hline 23 & 1967 & LONDERO et LAUDA & Brésil & $M$. laryngeus \\
\hline 24 & 1970 & CRAIG et FAUST & Porto-Rico & $M$. laryngeus \\
\hline 25 & 1970 & $\begin{array}{l}\text { FARIAS, cité par TEUSCHER } \\
\text { et Collab. }\end{array}$ & Equateur & M. laryngeus \\
\hline 26 & 1970 & JUNOD et Collab. & $\begin{array}{l}\text { France - Patient ayant } \\
\text { séiourné aux Antilles }\end{array}$ & $\begin{array}{l}M . \text { laryngeus } \\
M . \text { laryngeus }\end{array}$ \\
\hline 27 & 1970 & HO THI SANG et Collab. & Martinique & M. laryngeus \\
\hline
\end{tabular}

insomnies prolongées, anxiété et amaigrissement.

L'examen du patient ne montre ni signes pulmonaires, ni fièvre. La toux s'accompagne parfois de crises d'asthme de longue durée, de dyspnée et de vomissements quand les Nématodes sont fixés à la face postérieure du pharynx. Le pourcentage d'éosinophiles est élevé (plus de 20 p. 100). La durée de l'affection peut être assez longue: de 5 mois et demi (ST-JOHN et collab., 1929) à 8 mois et demi (HOFFMAN, 1932).

Les lésions, peu importantes, sont, en général, celles d'unc laryngo-pharyngite avec formation et rejet de fausses membranes. Le nombre de couples ne dépasse pas 5 paires (LENT et PENNA, 1939).

L'infestation se fait, sans doute, par ingestion d'eau ou d'aliments souillés par les larves $\mathbf{L}_{i}$.

Le diagnostic est difficile à poser lorsqu'il s'agit d'un premier cas et, souvent, il n'est établi qu'à la suite de l'élimination des parasites au cours d'une forte quinte de toux. L'examen du fond de la gorge à l'abaisse langue avec une lampe frontale permet, en cas de localisa- tion haute, de mettre en évidence les Syngames. L'examen au microscope des selles et des crachats est également d'un grand secours et la découverte d'œufs à deux blastomères lève le doute.

Dans les régions d'endémicité, on devra penser à la mammomonogamose chaque fois que l'on remarque, chez l'homme, une toux nocturne, intense, chronique et rebelle à tout traitement: l'absence de signes profonds, l'examen du fond de la gorge, la présence d'œufs caractéristiques dans les selles ou les crachats, assurent le diagnostic différentiel des autres affections pulmonaires.

Le pronostic est bénin, en dépit des désagréments physiques et psychologiques que la Syngamose provoque.

Le traitement consiste à extirper à la pince les parasites implantés dans la gorge.

Récemment (JUNOD et collab. 1970), on a préconisé l'administration de Thiabendazole par la voie buccale. La dose totale est de 9 grammes répartis ainsi : 2 grammes le premier jour, et un gramme par jour les sept jours suivants. Les résultats sont favorables et l'amélioration est sensible dès le troisième jour. 
Le Tétramisole lévogyre dont l'action sur les Nématodes bronchiques des ruminants est bien connue mériterait, à la dose de $3-4 \mathrm{mg} / \mathrm{kg}$. d'être expérimenté dans le traitement de la syngamose humaine.

En matière de prophylaxie, on ne peut que recommander des mesures hygiéniques classiques : cuisson des légumes et stérilisation de l'eau de boisson (CRAIG et FAUST, 1970).

\section{CONCLUSIONS}

Les auteurs étudient la mammomonogamose des ruminants domestiques et sauvages, affection due à la présence dans les voies respiratoires supérieures de Mammomonogamus laryngeus (RAILLIET, 1899) et de Mammomonogamus nasicola (VON LINSTOW, 1899) qui sont redécrits.

Mammomonogamus laryngeus est une espèce parasite du buffle, du bœuf et du zébu du sud-est asiatique. Mammomonogamus nasicola, outre les espèces précédentes, affecte les petits ruminants, certains Cervidés et le Buffle sauvage (Syncerus caffer). C'est un Nématode propre à l'Afrique, aux Antilles et - semblet-il - à l'Amérique du Sud.

Mammomonogamus okapiae est spécifique de l'Okapi en Afrique centrale.

Les symptômes sont discrets et se traduisent par des quintes de toux, rares et violentes, aboutissant parfois, chez les ovins, à l'asphyxie et à la mort.

Les parasites provoquent une inflammation catarrhale des muqueuses nasales (surtout $M$. nasicola), laryngo-pharyngées et trachéales.

Le diagnostic ante mortem est possible par la mise en évidence à l'examen coproscopique d'œufs ellipsoïdes (76-98 $\mu$ sur $38-60 \mu$ ), à coque épaisse et renfermant, en général, deux blastomères.

A l'autopsie, les Syngames se présentent comme des "filets de sang coagulé » qui nécessitent, après louverture de l'arbre trachéobronchique et des cavités nasales, un examen attentif et minutieux, ce que l'on oublie souvent.

La maladie, dont on ne peut actuellement chiffrer l'incidence économique, semble, en réalité, plus répandue qu'il n'apparaît au premier abord.

L'homme peut également en être atteint et l'on connaît aujourd'hui 25 cas de syngamose humaine aux Antilles, en Amérique du Sud et aux Philippines.

\section{REMERCIEMENTS}

Les auteurs tiennent à remercier vivement Madame GAUTHEY qui a bien voulu se charger d'une partie de l'iconographie.

\section{SUMMARY}

\section{Syngamosis of domestic and wild animals}

The authors make a survey of the present knowledges concerning the syngamosis of domestic and wild ruminants

This disease affects cattle, buffalo, zebu, sheep, goat, south american. deer, african game species of families Giraffidae and Bovidae and also the man in endemic areas (South America; West Indies; South East Asia).

They describe again the two main species: Mammomonogamus laryngeus (RAlLLIET, 1899) and Mammomonogamus nasicola (VoN Linstow, 1899).

They emphasize on the necessity, in tropical countries, of examining carefully the faeces for the presence of Syngamid eggs and, post-mortem, the upper respiratory tract and the nasa] passages to which the parasites are firmly attached.

\section{RESUMEN}

La mammomonogamosis de los rumiantes domésticos y salvajes

Los autores exponen los conocimientos actuales sobre la mammomonogamosis de los rumiantes doméstıcos y salvajes, enfermedad que ocurre 
en en buey, el búfalo, el cebú, la oveja, la cabra, cérvidos sudamericanos, bovinos y jirafas africanos, asi como en el hombre en las zonas de endemicidad (Asia del sudeste, Antillas y America del sur).

Describen de nuevo las dos especies principales:

Mammomonogamus laryngeus y Mammomonogamus nasicola.

Insisten sobre la necesidad, en ambiente tropical caldo y húmedo, de hacer sistematicamente examenes coproscopicos y autopsias completas en lo concerniente a las vías aéreas superiores y las cavidades nasales donde se fijan habitualmente los Mammomonogamus.

\section{BIBLIOGRAPHIE}

AMARAL (A. D. F.), QUAGLIA (F. O.), INOUE (C. R.), Sôbre novo caso de singamose humana. Revta. Med., 1954, 38, 209, 1-9.

BAER (J. G.), Etude critique des parasites de l'Okapi, Acta trop., 1950,7 (2) : 164-92.

BAKER (D. W.), Mal de Caderas or severe parasitism, Vet Med., 1951, 46, 11: 462

BAYLIS (H. A.), The fauna of British India including Ceylon and Burma. Nematoda. Vol, I: Ascaroidea and Strongyloidea, London, Taylor et Francis, 1936, $408 \mathrm{p}$.

BHALERAO (G.D.), Helminth parasites of the domestic animals in India, Monographie $\mathrm{n}^{0} 7$, $1935,287$.

BHATTACHARJEE (J.), A check-list of the Nematode parasites of the domesticated animals in Burma, Indian J. vet. Sci., 1937, 7 (2) : 87-96.

BHATTACHARJEE (M. L.), Bionomics of free living stages of Syngamus laryngeus (Railliet, 1899) a fairly common parasite in the larynx of cattle and buffaloes, Indian J. anim. Hith., 1966, 5 (2): 143-45.

BRUMPT (E.), Précis de Parasitologie, Paris, Masson, 1949, t. I : 882.

BUCKLEY (J. J. C.), On Syngamus nasicola Linstow, 1899 from sheep and cattle in the West Indies, J. Helminth., 1934, 12 (1): 47-62.

BWANGAMOI (O.), Helminth parasites of domestic and wild animals in Uganda, Bull, epizoot. Dis. Afr., 1968, 16 (4) : 433.

CABALLERO (C. E.), VOGELSANG (E. C.), Fauna helminthologica Venezolana. III. Algunos Nematodos de animales silvestres, Revta. Med. vet. Parasit., Caracas, 1950, 9: 54-67.

CHANDLER (A. C.), READ (P.C.), An introduction to parasitology, 10th ed., New York, London, Wiley and Sons, 1961: 443.

CHAPIN (E. A.), The spicules of Syngamus laryngeus, Parasitology, 1924, 11: 106.

CRAIG, FAUST (E.C.), Clinical Parasitology, 8th ed., Philadelphia, Lea and Febiger, 1970, pp. 318-20.

DE DOES (K.J.), Berchrijning van eenige dierlijke parasitien, Geneesk. Tijdschr. Ner-Ind., 1970, 28 : 263-79.

DROZDZ (J.), MALCZEWSKI (A.), Endoparasites et maladies parasitaires des animaux domestiques du Vietnam, Monogr. Lab. Par. Acad. Pol. Sci. Varsovie, 1967: 148-50.

ENDREJAT (E.), Helminths and helminthic diseases in Assam, Indian vet. J., 1964, 41 (8) : 538-42.

EUZEBY (J.), Les maladies vermineuses des animaux domestiques, t. I, fasc. 2, Maladies dues aux Nemathelminthes, Paris, Vigot frères, 1963, pp. $447-$ 48.

FROGER (P.E. J.), La syngamose. Thèse Méd. vét. Paris, 1930, 62 p.

FRONT (J. H.), Syngamus laryngeus in man. Report of three additionnal cases from Puerto-Rico, Boln. Asoc. Med. P. Rico, 1943, 35 (9) : 331-33.

GHESQUIERE (J.), Sur la répartition géographique de deux vers Syngames observés au Congo Belge, C.R. Somm. Séanc. Soc. Biogéogr., 1934, 89 : 10-2. GIOVINE (D.), Le piu communi malattie infettive e parassitariae del bestiame della Columbia, Nuovo ÉrCl, 1932, 37 (5): 89-96 et (6) : 101-07.

GOMES DE MORAES (R.), Parasitismo humano pelo Syngamus laryngeus, Archos. Bras. Med., 1947, 37 (3-4) : 97-101.

GRABER (M.) et Collab., Les Mammomonogamus des ruminants domestiques et sauvages (à paraître).

GRETILLAT (S.), Rapport de mission aux Antilles françaises, Monographie J.E.M.V.T. - I.N.R.A., 1966. pp. 34-37.

HALL (M. C.), A note on Syngamus laryngeus from cattle in the Philippine islands, Am. J. Vet. Med., 1915, 10, 395-96.

HALL (M. C.), Apparent atrophy of spicules with increasingly close and permanent union of the male and the female Syngamus, Parasitology, $1920,7: 100$.

HOFFMAN (W. A.), Gapeworm infestation in man, Puerto Rico J. Pub. Hith. trop. Med., 1931, 6: 381-3.

HOFFMAN (W. A.), Gapeworm infestation of man, Bo!n. Asoc. Méd. P. Rico, 1932, 24 (207): 703-4.

HO THI SANG, JUNOD (C.), PHILBERT (M.), Notes parasitologiques sur Syngamus laryngeus Railliet, 1899 et la syngamose humaine. A propos d'un cas de Syngamose bronchique chez l'homme, Bull. Soc. Path. exot., 1970, 63 (4) ; 488-97.

HOUDEMER (E.), TRAN THO-HUY, Syngame laryngien des bovidés au Tonkin, Bull. Vét. Aux. Indochine, 1923: 151-52.

HOUDEMER (E. F.), Recherches de parasitologie comparée indochinoise, Paris, Le François, 1938: 125.

JESUS (Z. DE), External and internal Metazoan parasites of Philippine cattle, Philipp. J, anim. Ind., 1938, 5 (1) : 21-34.

JORGE DA SILVA (A, A.), Alguns Nematodios de animais domesticos no estado da Bahia, Atas. Soc. Biol. Rio de J., 1961, 5 (3) : 10-13.

JUNOD (C.), PHILBERT (M.), HO THI SANG, Une observation de syngamose humaine à localisation bronchique. Premier cas traité et guéri par le Thiabendazole, Bull. Soc. Path. exot., 1970, 63 (4) : 483-88.

LAPAGE (G.), Veterinary Parasitology, Edimburg and London, Oliver Boyd, 1967: 138.

LEIPER (R. T.), Observations on certain helminths of man, Trans. R. Soc. trop. Med., 1913, 6 (8): 265-97.

LEIPER (R. J.), Syngamus kingi, a second case in man, Trans. R. Soc. trop. Med. Hyg., 1925, 19 : 279.

LENT (H.), PENNA (M.), Novo caso de singamose em homon no Brasil, Hospital, Rio de J., 1939, 16 (1) : 111-8.

LEVINE (N.D.), Nematode parasites of domestic 
animals and of man, Burg, Pub. Comp., 1968 , pp. 157-58.

LIMA DE (L. I.), BARBOSA (F. S.), Consideracoes em torno de um caso de Singamose humana, Récife, Publçoes Avuls. Inst. Aggeu Magalhaes, 1952, 1 : 27-34.

LINSTOW (O. VON), Nematoden aus der Berliner zoologischen sammlung, Mitt. Zool. Mus. Berl, 1899,1 : $5-26$.

LONDERO (A.T.) LAUDA (P.), Infeccăo humana por Syngamus laryngeus, Hospital, Rio de J., 1967, 72 (4) : 1267-69.

MACHADO (O.), Sobre Syngamus laryngeus. Caracteres dos exemplares encontrado em um caso de Singamose no homon, Boln. Inst. Vital, Brasil, 1938, 22 : 61-68 (description des exemplaires provenant de l'observation de Mello et Mello (1938).

MELLO (M.), MELLO (S.), Syngamus laryngeus no homen, Rev. Fluminense Med., 1938, 3: 457-60.

MOHAN (R. N.), Diseases and parasites of buffaloes. Part III: Parasites and miscellaneous diseases, Vet Bull. Weybridge, 1968, 38 (11): 740-41.

MOHAN (R. N.), GOTTS (M. G), Diseases and parasites of the african buffalo (Syncerus caffer), Vet. Bull. Weybridge, 1970, 40 (3) : 157-65.

MONTESTRUC et Collab, A propos d'un cas de syngamose humaine. Soc. Path exot, séance du 9 mai 1945 (non publié).

MUDALIAR (S. V.), ALWAR (V.S.), A check-list of parasites (Class Nematoda) in the department of parasitology, Madras veterinary college laboratory, Indian vet. J., 1947, 24 (2): 77-94.

NEVEU-LEMAIRE (M.), Traité d'helminthologie médicale et vétérinaire, Paris, Vigot frères, 1936, pp. 974-75.

ORR (W.), Observations on the occurrence of animals parasites, Rep. Vet. Dept. Malaya for 1937, 1938, pp. $93-100$.

PASSOS (N.), CASTRO BARBOSA (N.), Syngamus laryngeus na specie humana, Revta. Bras. Med., 1948,5 (5) : 340.

PATNAIK (M. M.), A note on bovine syngamosis, Indian vet. J., 1963, $40: 272-74$.

PINTO (C.), ALMEIDA (J. LINS DE), Synopsis des helminthes parasites des animaux domestiques du Brésil, Comm. 30 Jub. Prof. Skrjabin et 15e Ann. Inst. Helm. U.R.S.S., Moscou, 1937 : 469-72.

RAILLIET (A.), Syngame laryngien du bœuf, C.R. Séanc. Soc. Biol, 1899,6 (10): 174-76.

RAILLIET (A.), Les helminthes des animaux domestiques et de l'homme en Indochine française, $B u l l$. Soc. Zool. Fr., 1925, 50: 14.

RANSOM, Bur Anım. Ind., 1921 (939) (cité par Froger).

RESSANG (A. A), FISCHER (H.), MUCHLIS (A.), The indonesian veterinarian : his education, activities and problems, Communicationes vet., 1959, 2 (3) : $55-99$

ROSE ROSETTE, Un cas de syngamose laryngienne chez le mouton, Bull. Soc. Path. exot., 1934, 27 (3) : 264-65.
RYZHIKOV (K. M.), Phylogenic relationship of Nematodes of the family Syngamidae and an attempt to reconstruct their systematics (en russe), Dokl. Akad. Nauk. S.S.S.R., 1948, 62 (5) : 733-36.

SACHS (R.), SACHS (C.), A survey of parasitic infestation of wild herbivores in the Serengeti region in Northern Tanzania and the lake Rukwa region in Southern Tanzania, Bull. epizoot. Dis. Afr., 1968, 16 (4) : 459-60.

SACHS (R.), DEBBIE (J. G.), A field guide to the recording of parasitic infestation of game animals, E. Afr. Wild. J., 1969, $7:$ 31-32.

SACHS (R.), FRANK (H.), BINDERNAGEL (J. A.), New host records for Mammomonogamus in African game animals through application of a simple method of collection, Vet. Rec., 1969, 84 (22) : 562-63.

SAINT-PRIX (L.), Sur la syngamose humaine dans le département de la Martinique, Ann/s. Parasit. hum. comp., 1950, 25 (3) : 235-36.

SENEVIRATNA (P.), MAHALINGAM (S.), Some helminths hitherto not reported from Ceylon, Ceylon vet. J., 1962,10 (2-3) : 88-92.

SHEATHER (A. L.), SHILSTON (A.W.), Syngamus laryngeus in cattle and buffaloes in India, Bull. Agric. Res. Inst. Pusa, 1920 (92) : 1-8.

SKRJABIN (K. I.) et Collab., Key to Parasitic Nematodes. Vol. III : Strongylata, Jerusalem, Israel Prog Trans. 1961, pp. 187-90.

SMIT (H. J.), Parasitologische studien in Niederländishchr-Indien, Dt. Tieràrztl. Wschr., 1922, 30 : $506-12 ; 526-31 ; 546-48$.

SOULSBY (E. J. L.), Textbook of veterinary clinical parasitology, Oxford, Blackwell Sci. Pub., 1965, 747

ST JOHN (J.H), SIMMONS (J.S.), GARDNER (L. L.), Infestation of the lung by a Nematode of the genus Cyathostoma, J. Am. Med. Ass., 1929, 92: 1816-18.

TEUSCHER (E.) et Collab., Le diagnostic coprologique de la Syngamose bovine en Equateur avec une note sur l'efficacité probable du Tétramisole, Zentbl. Vet. Med., 1970, 17 (2) : 324-30.

THERY (A.), Les Syngamoses, thèse Méd. vét. Paris, $1962, n^{0} 38,74$ p.

TRAVASSOS (L.), Notas helminthologicas, Braz.Med., 1921, $35(6): 67$.

VAN DEN BERGHE (L.), Contribution à l'étude des parasites de l'Okapi, Rev. Zool. Bot. Afr., 1937, $29: 141-50 ; 361-9$.

VAZ (Z), Sobre a pretensa validez de Syngamus nasicola. Presensa do Syngamus laryngeus nas fossas nasaes de carneiros do Brasil, Archos Inst. biol., S. paulo, 1935,6 : 35-39.

WARE (F.), Two uncommon Nematode parasites of cattle, J. comp. Path., 1925, 38 (2) : 86-89.

WELLS (A. M.), Identity of hookworm in the throat, Br. Med. J., 1951, 1, 4712, 952.

YAMAGUTJ (S.), Systema helminthum. Vol. III : the Nematodes of vertebrates, Part I, 1961, 531-32. 